\title{
Éthique \\ et prélèvements \\ d'organes \\ sur donneurs \\ décédés après \\ arrêt cardiaque
}

Corinne Antoine, Alain Tenaillon

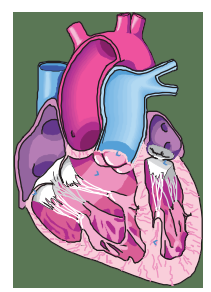

L'article de J.C. Tortosa et al. paru dans le numéro de février 2010 de Médecine/Sciences $(\rightarrow)$ [1] aborde de façon claire et avec un point de vue personnel quelques-uns des principaux problèmes éthiques soulevés

$(\rightarrow)$ voir $\mathrm{m} / \mathrm{s} \mathrm{n}^{\circ} 2$, février 2010, p. 209 par la pratique des prélèvements d'organes réalisés sur des patients décédés après arrêt cardiaque. Comme le souligne bien l'article, certains problèmes éthiques sont différents selon que le donneur potentiel fait un arrêt cardiaque de façon «programmée » dans un service de réanimation en raison d'une décision d'arrêt des thérapeutique actives (classe III des critères de Maëstricht), pratique qui concerne essentiellement les États-Unis, la Grande-Bretagne, les Pays-Bas et la Belgique, ou s'il fait cet arrêt de façon spontanée dite «non programmée » en présence (classe II) ou en l'absence (classe I) d'une équipe de secours, pratique qui concerne alors essentiellement la France et l'Espagne. Nous voudrions cependant apporter sinon un point de vue différent, du moins quelques précisions sémantiques ou techniques et une interprétation éthique complémentaire.

- Le choix délibéré du Dr Tortosa d'utiliser le terme devenu classique de «donneur à cœur arrêté » peut être mal compris et créer un doute quant à la réalité de la mort, ambiguïté qui persiste tout au long de l'article. Après une longue réflexion, l'Agence de la biomédecine a banni cette appellation pour lui préférer celle de « donneur décédé après arrêt cardiaque » (DDAC), de façon à clairement indiquer que la règle du donneur décédé doit être appliquée avant de parler de donneur, et que le décès doit être constaté avant toute démarche technique à visée de prélèvement d'organe (la même démarche a été faite pour le donneur en état de mort encépha-

lique appelé dorénavant «donneur décédé en mort encéphalique», DDME).

- Le deuxième point concerne le terme d'arrêt cardiaque « irréversible » pour définir la mort d'un sujet. À nouveau, ce terme est impropre car la mort, dans la plupart des pays, est actuellement définie par la destruction des structures encéphaliques; le caractère irréversible ou non de l'arrêt cardiaque n'a donc que peu d'importance, ce qui compte est la durée de l'insuffisance et de l'arrêt de la perfusion cérébrale qui vont être responsables d'une destruction, elle irréversible, des structures encéphaliques. II n'y a donc pas de paradoxe à avoir dans certains cas un décès survenu à la suite d'un arrêt cardiaque par destruction encéphalique et la possibilité d'avoir un cœur non battant quoique encore 
fonctionnel, pouvant être prélevé pour être greffé. De fait, le décret définissant la mort dans le cadre des prélèvements d'organes en France ne parle pas d'arrêt cardiaque irréversible mais d'arrêt cardiaque et respiratoire «persistant », terme beaucoup plus en accord avec la réalité clinique et qu'il convient pour nous de privilégier.

- Le troisième point concerne, dans le cadre des arrêts cardiaques programmés, la durée indispensable de l'arrêt pour affirmer que la règle du donneur décédé est respectée, et que la procédure de prélèvement ne se déroulera pas sur un sujet encore vivant. Dans la réalité clinique, lors d'un arrêt des thérapeutiques actives et notamment de la ventilation mécanique, il existe une phase plus ou moins longue avant que ne survienne l'arrêt cardiaque et pendant laquelle la perfusion cérébrale est de très mauvaise qualité en raison d'une ventilation insuffisante sans complément d'oxygène et d'une hypotension progressive par baisse fonctionnelle de l'activité cardiaque. Cette période d'hypoperfusion cérébrale majeure est en grande partie responsable de la détérioration des structures cérébrales, dès lors un arrêt cardiaque effectif de très faible durée est suffisant pour compléter la destruction définitive de l'encéphale. II faut cependant reconnaître qu'il est difficile d'admettre que chaque équipe puisse donner sa propre durée alors que l'Institut de médecine des États-Unis a fixé cette durée à 5 min à l'occasion de recommandations itératives [2-4].

- Le quatrième point concerne à nouveau la notion «de doute sur le statut vital du donneur ». Ce point est particulièrement complexe car il est impossible de «dire » le moment de la mort; en effet, mourir est un continuum et toutes les cellules de l'organisme ne sont pas détruites au même instant. La notion de mort est donc un concept anthropologique, philosophique, métaphysique voire juridique qui dépend de chaque société. En revanche, les progrès de la connaissance médicale ont permis d'identifier le moment où l'on peut considérer que le processus qui aboutira à la mort est irréversible: il s'agit actuellement du moment où les critères neurologiques de mort encéphalique sont tous présents. Dans le cas des catégories I ou II de Maëstricht, le doute sur le statut vital du sujet est, dans ce contexte, purement théorique. Le contrôle de la mort sur le plan technique est assuré non pas par la durée de l'arrêt cardiaque mais sur l'inefficacité de la ressuscitation, comme le confirme tout au long de ces manœuvres l'absence de tout signe d'activité du tronc cérébral et donc de vie. Il s'agit de critères cliniques objectifs, traduisant la mort du tronc cérébral, recherchés à plusieurs reprises au cours de la ressuscitation, confirmés durant les 5 min d'arrêt de toutes les manœuvres de ressuscitation, et permettant d'affirmer le décès. Ces critères sont bien définis en France dans le décret de 1996 précisant les conditions de la mort en cas de prélèvement d'organe. En cas de doutes sur la persistance de signes même minimes d'activité du tronc cérébral, un algorithme clair a été défini pour éventuellement pouvoir utiliser des manœuvres complémentaires de sauvetage comme la circulation extracorporelle d'assistance. II est, dans ce contexte, faux de laisser croire que les manœuvres utilisées après la constatation du décès pour préserver les organes pourraient, en réoxygénant le cerveau, faire réapparaître une activité cérébrale; cela signifierait que la destruction encéphalique n'est pas avérée et que le sujet n'est pas mort, à nouveau le doute sur la mort !

- Le cinquième point est celui du conflit d'intérêt souligné régulièrement dans ce contexte. Ce problème peut être soulevé pour la majorité des actes thérapeutiques en médecine, ce qui n'empêche pas les malades de se faire soigner! Dans le contexte des prélèvements d'organe, des garde-fous et des protocoles clairs de séparation entre les équipes soignantes et les équipes impliquées dans le prélèvement et la greffe ont été mis en place dans nos différents pays. Il faut arrêter de penser que les médecins sont dépourvus de conscience professionnelle; il faut rappeler que les équipes de réanimation, qu'elles soient mobiles (SMUR, services médicaux d'urgence) ou intrahospitalières, se battent d'abord et toujours pour la survie du malade pris en charge et que la mise en œuvre d'une procédure de prélèvement, loin de soit-disant valoriser leur échec, complique toujours beaucoup plus la vie de l'équipe que de simplement constater la mort d'un malade en évitant tout prélèvement.

- Le sixième point est celui du problème éthique soulevé par l'absence de consentement, du sujet avant son décès ou de ses proches, avant la mise en œuvre rapide de techniques de préservation des organes. Ce choix est basé sur le principe du consentement présumé en vigueur en France et s'exerce dans les mêmes conditions que chez les sujets en état de mort encéphalique où il ne semble pas poser de question majeure.

- Le dernier point que nous voulions aborder est celui évoqué du « manque de transparence vis-à-vis des familles». Ce point est fondamental car on sait que de la confiance du public dépendent tous les programmes encadrant les dons d'organes et que le contexte d'une mort qu'elle soit programmée, Maëstricht III, ou brutale et inattendue, Maëstricht I ou II, pour les DDAC, mais aussi pour les DDME, reste pour les proches une circonstance dramatique au cours de laquelle toute intervention extérieure peut être considérée comme une agression et une intrusion dans un champ de l'intime totalement protégé. Dans ce contexte transgressif, toute attitude non transparente, sous prétexte de paternalisme ou de volonté délibérée de prélèvements d’organe, est particulièrement mal venue. Aussi, après les premières expériences d'approches des familles dans ce contexte de DDAC non programmé en 
France, l'attitude s'est clarifiée. Un groupe de travail, mis en place par l'Agence de la biomédecine avec les professionnels de terrain, a opté récemment pour une transparence absolue et pour demander aux équipes de SMUR ou d'urgence en charge de la ressuscitation de ces malades, de n'envisager le transfert en vue de prélèvement d'organe lorsque des proches sont présents au moment de la constatation du décès, que si ces derniers ont témoigné de la non-opposition du défunt. $\diamond$

Ethics and organ retrievals on deceased donors

after cardiac stop

\section{CONFLIT D'INTÉRÊTS}

Les auteurs déclarent n'avoir aucun conflit d'intérêts concernant les données publiées dans cet article.

\section{RÉFÉRENCES}

1. Tortosa JC, Rodríguez-Arias Vailhen D, Moutel G. Questions éthiques soulevées par les deux types de protocoles de prélèvements d'organes à cœur arrêté : aspects particuliers à la France, l'Espagne et aux États-Unis. Med Sci (Paris) 2010 ; 23 : 209-13.

2. Bernat JL, D'Alessandro AM, Port FK, et al. Report of a national conference on donation after Cardiac Death. Am J Transplant 2006; 6: 281-91.

3. Institute of Medicine, National Academy of Sciences. Non-heart-beating organ transplantation: medical and ethical issues in procurement. Washington, DC : National Academy Press, 1997.

4. Institute of Medicine, National Academy of Sciences. Non-heart-beating organ transplantation: practice and protocols. Washington, DC : National Academy Press, 2000.

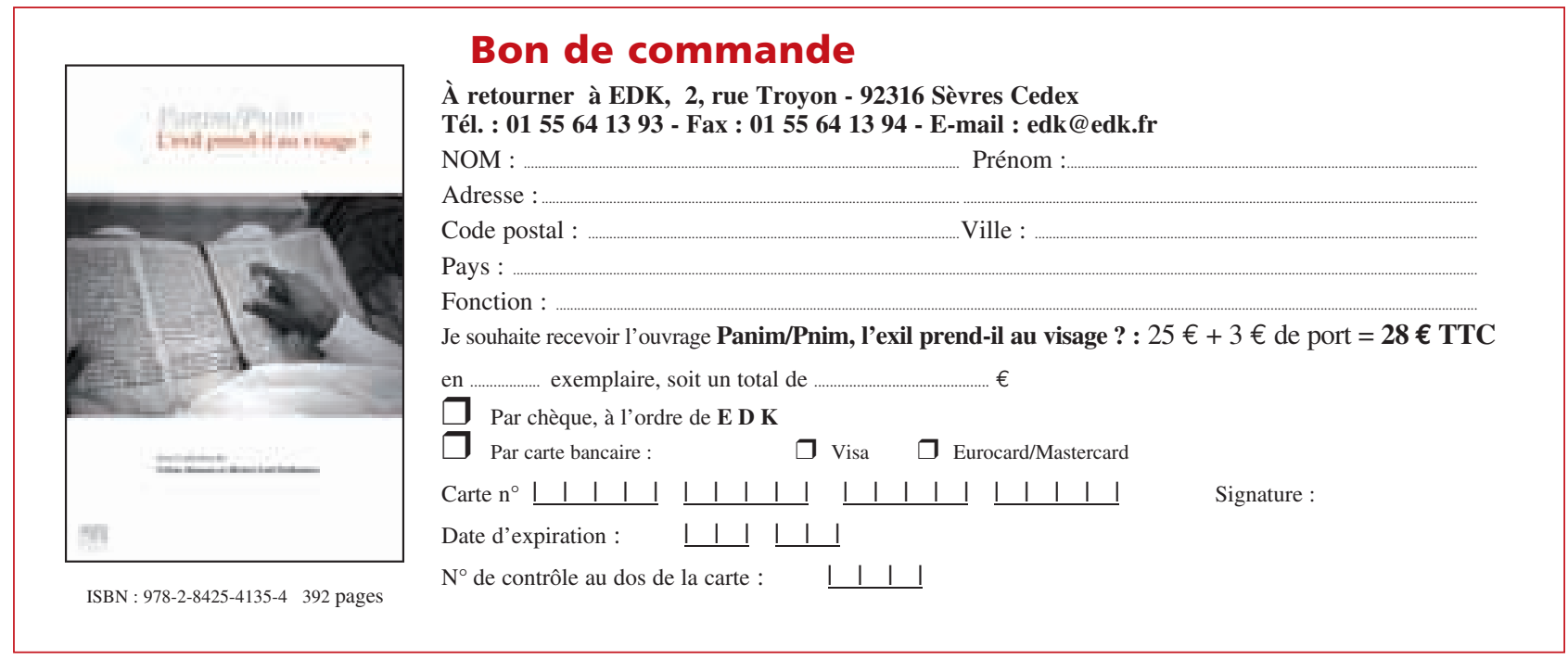

TIRÉS À PART

C. Antoine

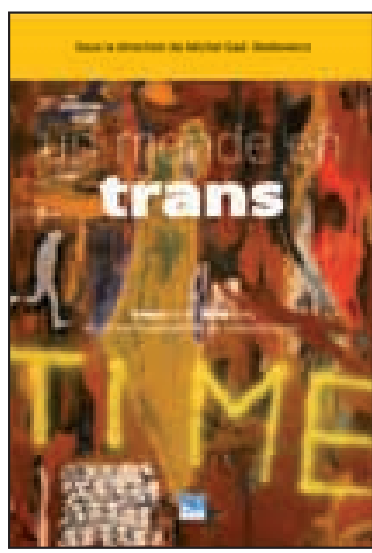

ISBN : 978-2-8425-4129-3 344 pages

\section{Bon de commande}

À retourner à EDK, 2, rue Troyon - 92316 Sèvres Cedex

Tél. : 0155641393 - Fax : 0155641394 - E-mail : edk@edk.fr

NOM : ............ Prénom .

Adresse :

Code postal :

Ville :

Pays :

Fonction :

Je souhaite recevoir l'ouvrage Un monde en trans : $24 €+3 €$ de port $=27 €$ TTC

en ................ exemplaire, soit un total de

$€$

$\square$ Par chèque, à l'ordre de $\mathbf{E} \mathbf{D} \mathbf{K}$

$\square$ Par carte bancaire : $\quad \square$ Visa $\square$ Eurocard/Mastercard

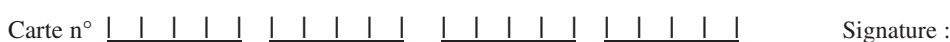

Date d'expiration: $\quad|\quad| \quad|\quad|$

$\mathrm{N}^{\circ}$ de contrôle au dos de la carte 